\title{
Necrotizing Scleritis and Peripheral Ulcerative Keratitis Associated with Wegener's Granulomatosis
}

\author{
Jianjun $\mathrm{Gu} \cdot$ Sheng Zhou $\cdot$ Ruxing Ding $\cdot$ \\ Wumaier Aizezi $\cdot$ Aixin Jiang $\cdot$ Jiaqi Chen
}

To view enhanced content go to www.ophthalmology-open.com

Received: May 20, 2013 / Published online: July 10, 2013

(C) The Author(s) 2013. This article is published with open access at Springerlink.com

\section{ABSTRACT}

Introduction: To evaluate the complications, efficacy of medical and surgical treatment, and outcome in patients with necrotizing scleritis and peripheral ulcerative keratitis associated with Wegener's granulomatosis.

Methods: The authors reviewed a series of seven patients with Wegener's granulomatosis treated in the Corneal Department of Zhongshan Ophthalmic Center and the Department of Ophthalmology of Kashgar First People's Hospital. A detailed chart review was performed to determine demographic

J. Gu $\cdot$ S. Zhou $\cdot$ J. Chen $(\bowtie)$

State Key Laboratory of Ophthalmology, Corneal Department, Zhongshan Ophthalmic Center, Sun Yat-sen University, 54 Xianlienan Road, Guangzhou 510060, People's Republic of China e-mail: docjiaqi@163.com

J. Gu $\cdot$ R. Ding $\cdot$ W. Aizezi · A. Jiang

Department of Ophthalmology,

Kashgar's First Hospital, 66 Yinbin Road, Kashgar 844000, People's Republic of China

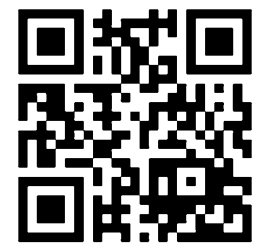

Enhanced content for this article is available on the journal web site: www.ophthalmology-open.com characteristics, ocular presentation, biopsy and laboratory testing results, treatment, and final outcome.

Results: Wegener's granulomatosis was indicated by ocular and/or systemic findings; biopsy and immunohistochemistry results supported the diagnosis. Patients with necrotizing scleritis and/or peripheral ulcerative keratitis received cytotoxic immunosuppressive therapy; this, in conjunction with surgical treatment, halted the relentlessly progressive inflammation and preserved the integrity of the globe in $78 \%$ of eyes. Best-Corrected Visual Acuity remained stable in four of nine eyes, was improved in two of nine eyes, and decreased in three of nine eyes (secondary to cataract and/or stromal scarring). Although one patient died, treatment with corticosteroids and cytotoxic agents dramatically improved outcomes in these patients.

Conclusion: Necrotizing scleritis and peripheral ulcerative keratitis often have a poor visual outcome, and may herald an underlying systemic vasculitis. Wegener's granulomatosis, with the associated necrotizing scleritis and peripheral ulcerative keratitis, should be managed with aggressive immunosuppression 
to avoid the associated morbidity and mortality. Thus, the ophthalmologist may play a significant role in its early diagnosis and treatment.

Keywords: Antineutrophil cytoplasmic antibodies; Biopsy; Granulomatous lesions; Immunosuppressive agents; Necrotizing scleritis; Peripheral ulcerative keratitis; Wegener's granulomatosis

\section{INTRODUCTION}

Wegener's granulomatosis (WG) is characterized by necrotizing granulomatous lesions of the upper and lower respiratory tract, together with a necrotizing glomerulonephritis and widespread disseminated vasculitis. The form of WG is designated "limited" when only one or two organ systems are involved without renal involvement [1]. Cases have been reported of patients having limited forms of WG with involvement of the respiratory tract, skin, palatal, ear, and salivary gland [2, 3]. Ahmed et al. [4] reported cases of very limited WG, having only ocular involvement and no respiratory or renal disease. Untreated $W G$ is rapidly fatal, with renal failure being the primary cause of death. In the localized form of WG, the absence of renal involvement reduces the mortality rate [2]. Onset of renal disease is associated with a mean survival of 5 months; $82 \%$ of patients die within 1 year, and more than $90 \%$ die in 2 years [5]. Harper et al. [6] reported patients with limited WG who progressively evolved to systemic WG over the course of follow-up. This concept of progressively evolving WG with increased organ involvement is important, since timely therapeutic intervention can prevent the otherwise probable morbidity and mortality.
The diagnosis of limited forms of WG can be difficult. Early identification of the limited ophthalmic variant has been based on a combination of histopathologic findings and positive immunolabeling with cytoplasmic antineutrophil cytoplasmic antibodies (cANCA).

The therapeutic outcome of ophthalmic complications of WG in the USA and Europe has significantly improved over recent years [7], largely as a result of earlier diagnosis and treatment with a combination of glucocorticoids and cyclophosphamide (CP); however, there is a dearth of publications concerning the natural history, diagnosis and treatment of WG in Asian populations [8]. To rectify this deficiency, the authors review the clinical features, management, and visual outcomes of seven cases with necrotizing scleritis (NS) and/or peripheral ulcerative keratitis (PUK) associated with WG in a Chinese population.

\section{METHODS AND PATIENTS}

A review of the records of patients with scleritis seen in the Corneal Department of Zhongshan Ophthalmic Center between 2005 and 2012, and the Department of Ophthalmology of Kashgar First People's Hospital between 2009 and 2012 was performed. The Ethics Committees of Zhongshan Ophthalmic Center and Kashgar First People's Hospital approved this retrospective study, which has been conducted according to the principles expressed in the Helsinki Declaration of 1975, as revised in 2000 and 2008. The Ethics Committees specifically waived the need for consent.

A comprehensive review of patients documents was performed. Best-corrected visual acuity (BCVA) was measured by means of 
a Snellen chart. A detailed ophthalmic examination, including testing of corneal touch-sensitivity, slit-lamp examination, ocular pressure, and fundus examination, was performed and the site and extent of pathological changes in the sclera and associated corneal regions were determined. The laboratory evaluation included complete blood count, erythrocyte sedimentation rate (ESR), rheumatoid factor (RF), antinuclear antibodies, complement fixation, circulating immune complexes, cANCA, C-reactive protein (CRP), renal function studies, urinalysis, sinus and chest radiographs, Venereal Disease Research Laboratory (VDRL) testing, and TB skin testing that was judged to be positive if the extent of induration was $\geq 10 \mathrm{~mm}$. Conjunctival, corneal, and/or scleral biopsy samples were collected at the time of ocular surgery to search for any evidence of granulomatosis or vasculitis, and scrapings and cultures were analyzed by Gram- and Giemsa-staining.

Local ocular therapy consisted of topical dexamethasone and antibiotics (tobramycin and dexamethasone), topical cyclosporine (cyclosporin A), mydriatics, and artificial tears. Corticosteroids (dexamethasone and prednisone) and immunomodulatory/ cytotoxic drugs [methotrexate (MTX) and CP] were administered systemically, orally, or intravenously. The drug dosage was modified to obtain a good therapeutic effect with minimal complications of cytotoxic agents, such as declining leukocyte count.

\section{RESULTS}

A review of the records of 145 patients with scleritis seen in the Corneal Department of Zhongshan Ophthalmic Center between 2005 and 2012, turned up six cases identified with positive WG tests. One additional patient with similar findings was identified among 32 patients with scleritis in the Department of Ophthalmology of Kashgar First People's Hospital between 2009 and 2012. Of the 177 patients with scleritis, diffuse anterior (112 cases) was the most common type of scleritis observed in this study population, followed by nodular (31 cases), necrotizing (26 cases), and posterior scleritis (8 cases). Demographic data for the seven patients are summarized in Table 1. There were five men and two women, with an average age of 52 years (range 30-65 years). Five patients had unilateral sign of NS or PUK and two had bilateral sign of NS or PUK. Five patients had the limited form of WG, while two had the complete form. Redness and aching pain in the eyes were the common presenting complaints. At presentation, five cases had corneal involvement (sclerokeratitis), and two had NS. During 10 months of treatment, one developed glaucoma, one had choroidal detachment, and one had secondary cataract. BCVA was initially less than 20/40 in five cases (Table 2). Ocular lesions were the initial manifestations of WG in five of the seven cases. The duration of ocular signs before the diagnosis of WG ranged from 25 days for one patient with unilateral NS and PUK to 4 months for one patient with a unilateral peripheral corneal ulcer. These two eyes had been treated surgically before referral to the authors' hospital; the unilateral NS plus PUK was treated with a full-thickness corneal graft within 4 months, and the other with amniotic membrane transplantation within 3 months of the onset of disease symptoms.

Laboratory investigations revealed elevated ESR and positive cANCA in all seven patients. Rheumatoid factor was positive in case 3 . Chest $\mathrm{X}$-rays revealed pulmonary cavities and 
Table 1 Clinical features and treatment response

\begin{tabular}{|c|c|c|c|c|c|c|c|c|}
\hline \multirow[t]{2}{*}{ Case } & \multirow{2}{*}{$\begin{array}{l}\text { Age, } \\
\text { years/sex }\end{array}$} & \multirow[t]{2}{*}{ Eye } & \multirow{2}{*}{$\begin{array}{l}\text { Episodes of } \\
\text { ocular } \\
\text { disease }\end{array}$} & \multicolumn{3}{|c|}{ Immunosuppressive therapy } & \multirow{2}{*}{$\begin{array}{l}\text { Ocular } \\
\text { therapy }\end{array}$} & \multirow[t]{2}{*}{ Complications } \\
\hline & & & & Steroid & Cytotoxic & $\begin{array}{l}\text { Topical } \\
\text { cyclosporine }\end{array}$ & & \\
\hline \multirow[t]{2}{*}{1} & $54 / \mathrm{M}$ & OD & NS & Oral, IV & $\begin{array}{l}\text { CP, MTX } \\
\text { oral }\end{array}$ & + & SPG & Glaucoma \\
\hline & & OS & NS & & & + & SPG & $\begin{array}{l}\text { Graft melting that was } \\
\text { managed by } \mathrm{CP} \text {, } \\
\text { glaucoma }\end{array}$ \\
\hline 2 & $52 / \mathrm{F}$ & OS & NS, PUK & Oral, IV & $\mathrm{CP}$ oral & + & LK & Choroidal detachment \\
\hline 3 & $56 / \mathrm{M}$ & OD & NS, PUK & Oral, IV & CP oral & + & $\begin{array}{l}\text { LK with } \\
\text { intralamellar } \\
\text { patch }\end{array}$ & $\begin{array}{l}\text { Double anterior } \\
\text { chambers }\end{array}$ \\
\hline 4 & $65 / \mathrm{M}$ & OS & NS & Oral, IV & MTX oral & + & SPG & Cataract progression \\
\hline 5 & $54 / \mathrm{M}$ & OD & PUK & Oral, IV & $\mathrm{CP}$ oral & + & LK & \\
\hline 6 & $50 / \mathrm{F}$ & OD & NS, PUK & Oral, IV & CP oral & + & & \\
\hline 7 & $30 / \mathrm{M}$ & $\begin{array}{l}\text { OD } \\
\text { OS }\end{array}$ & $\begin{array}{l}\text { NS, PUK } \\
\text { NS, PUK }\end{array}$ & Oral & CP, IV & + & $\mathrm{BCL}$ & $\begin{array}{l}\text { Death on the } 102 \text { nd day } \\
\text { of admission }\end{array}$ \\
\hline
\end{tabular}

$B C L$ bandage contact lens, CP cyclophosphamide, $F$ female, $I V$ intravenous, $L K$ lamellar keratoplasty, $M$ male, $M T X$ methotrexate, $N K$ necrotizing scleritis, $O D$ right eye, $O S$ left eye, $P U K$ peripheral ulcerative keratitis, $S P G$ scleral patch graft + Topical cyclosporine treatment

Table 2 Clinical data

\begin{tabular}{llllllll}
\hline Case & Eye & Diagnosis & Serology $(+)$ & Initial BCVA & Final BCVA & Follow-up, months & Relapse \\
\hline 1 & OD & NS & cANCA & $20 / 100$ & $20 / 200$ & 35 & Lung granuloma \\
& OS & NS & cANCA & $20 / 50$ & $20 / 50$ & 35 & 15 \\
2 & OS & NS, PUK & cANCA & $20 / 200$ & $20 / 200$ & 21 & NS \\
3 & OD & NS, PUK & RF, cANCA & $20 / 100$ & $20 / 200$ & 14 & 26 \\
4 & OS & NS & cANCA & $20 / 40$ & $20 / 200$ & 10 & 4 \\
5 & OD & PUK & cANCA & $20 / 80$ & $20 / 40$ & $20 / 30$ & 4 \\
7 & OD & NS, PUK & cANCA & $20 / 40$ & $20 / 100$ & $20 / 100$ & \\
\hline
\end{tabular}

$B C V A$ best-corrected visual acuity, $O D$ right eye, $O S$ left eye, $N S$ necrotizing scleritis, $P U K$ peripheral ulcerative keratitis, $R F$ rheumatoid factor, $C A N C A$ cytoplasmic antineutrophil cytoplasmic antibody 
infiltrates in two patients (cases 6 and 7). X-rays in cases 1,2 , and 7 revealed mucosal thickening in the sinuses. Hematuria was seen in two patients (cases 1 and 7). Histological examination of nasal biopsies in cases 1 and 2 showed necrotizing giant cell granulomas with foci of inflammation. Subglottic stenosis of the pharynx was present in case 2 .

Surgery was required in five patients; two cases of NS received autologous sclera grafts, two cases of PUK were treated with lamellar keratoplasty, and one case of paracentral corneal perforation was repaired with a tectonic intralamellar patch plus an onlay graft.

In the patients with NS and/or PUK progressive inflammation was halted by the combination of immunosuppressive and surgical therapy in $78 \%$ of eyes. BCVA remained stable in four of nine eyes, was improved in two of nine eyes, and decreased in three of nine eyes (secondary to cataract and/ or stromal scarring).

\section{Selected Case Reports}

\section{Case 1}

A 54-year-old male patient had a 3-month history of NS with $>120^{\circ}$ extension in both eyes. The patient had been given topical steroids without effect before referral. At presentation, in addition to mild anterior uveitis and partial posterior iris synechiae, a perforation of the sclera was seen at the 3 and 9 o'clock meridians in the right eye, with disruption of the adjacent limbal arcade; and scleritis involved the lower half-circumference in the left eye, with high activity from 7 to 9 o'clock (Fig. 1a). BCVA was 20/100 in the right eye, and 20/50 in the left. Ocular pressure and fundus examination were normal in both eyes. Blood examinations disclosed elevations of serum CRP and ESR, and positive cANCA. The results of syphilis serology and tuberculin (TB) tests were negative. Urinalysis showed microscopic hematuria and proteinuria, but creatinine clearance was normal. The chest $\mathrm{X}$-ray was normal, but skull X-rays revealed mucosal thickening of the left maxillary sinus. Histopathology of nasal biopsy tissue revealed that the mucosa was infiltrated by lymphocytes, plasma cells, multinucleated giant cells, and epithelioid histiocytes.

The condition was diagnosed as WG, and treatment with systemic corticosteroids and MTX commenced. On October 13, 2005, a donor sclera graft was customized to the appropriate size and thickness, secured to the edges of the resection site of the left eye, and covered with a conjunctival flap. Histological analysis of the margin of the necrotic sclera showed necrotic collagen, inflammatory cells around the capillary walls, and granulomatous inflammation with lymphocytes, eosinophils, epithelioid cells, and multinucleated giant cells (Fig. 1b). Two weeks later, the conjunctival flap became retracted, leaving the scleral graft bare; the scleral graft developed lysis at 9 o'clock (Fig. 1c), and there were $1+$ aqueous cells, posterior iris synechiae, and elevated intraocular pressure in both eyes. Therefore, following consultation with a rheumatologist, the medical treatment was changed to corticosteroids and CP. The original scleral graft was re-sutured, and an amniotic membrane graft was applied; this arrested the lysis of the scleral graft, which was found to be vascularized 10 days later (Fig. 1d). The transplantation of the scleral graft in the right eye was uneventful (Fig. 1e, f). In both eyes, direct communication between the anterior and posterior chambers was established by Yttrium Aluminium Garnet (YAG) iridotomy to prevent the blockage of aqueous drainage by the progressive development of iris synechiae. 

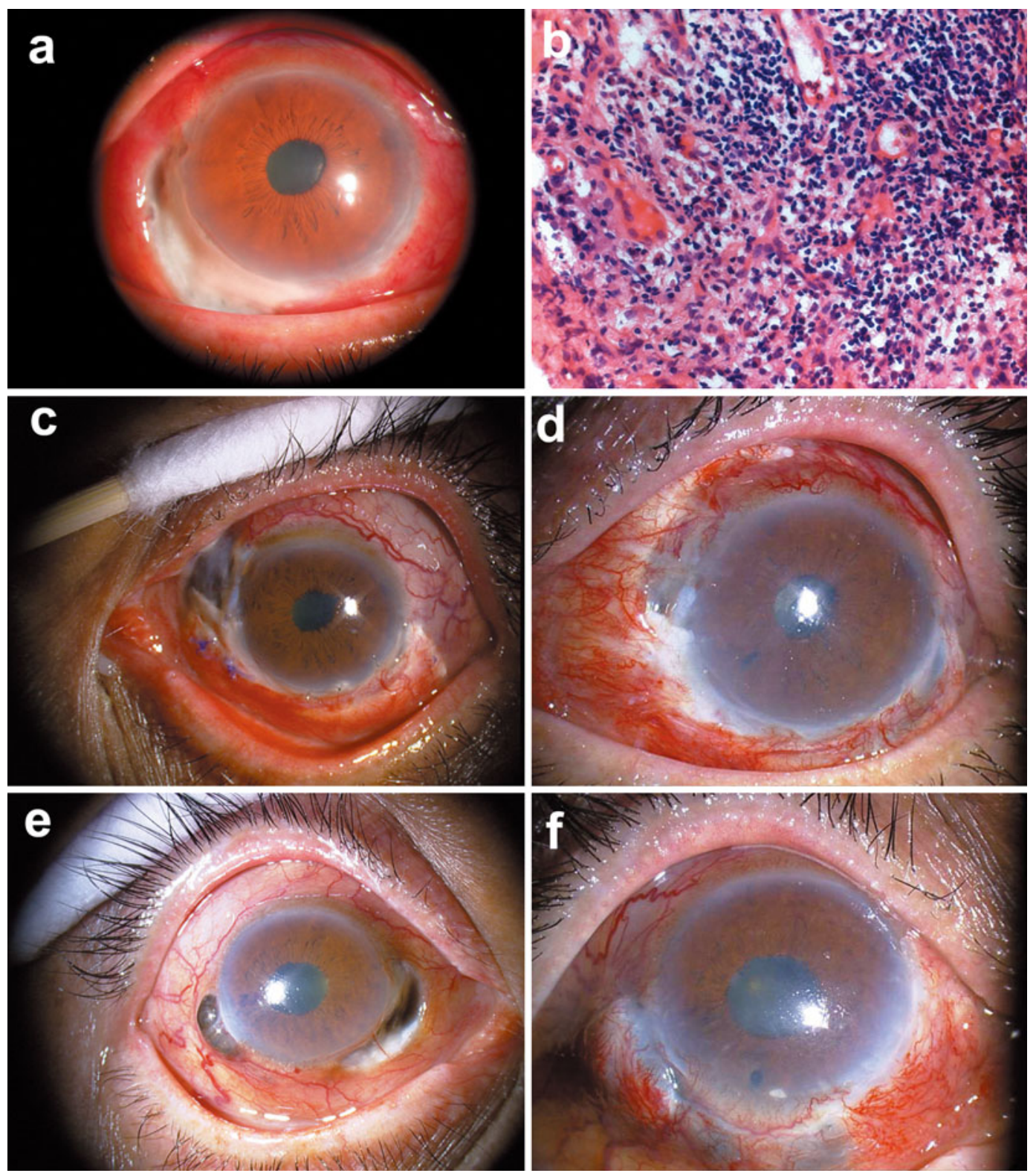

Fig. 1 Montage of clinical and histopathology illustrations (case 1). a Necrotizing scleritis in left eye of a 54-year-old man with Wegener's granulomatosis. b Scleral biopsy specimen from left eye illustrating collagen necrosis, and granulomatous inflammation with lymphocytes, eosinophils, epithelioid cells, and multinucleated giant cells (magnification $\times 400$; hematoxylin-eosin stain). c The conjunctival flap has developed retraction, leaving the

\section{Case 3}

A 56-year-old male patient with hyperlipidemia was referred for treatment of corneal melt. The patient complained of a recent episode of

scleral graft bare and revealing lysis of the graft in the left eye. $\mathbf{d}$ The lysis of the scleral graft was arrested and the vascularization of the graft was detected after amniotic membrane graft transplantation and systemic administration of cyclophosphamide. e Severe necrotizing disease in the same patient, with scleral thinning and perforation of the right eye. $\mathbf{f}$ Scleral graft performed after medical control of disease activity

redness and photophobia in the right eye. Ocular history included corneal perforation in the left eye treated by penetrating keratoplasty at another hospital 9 months earlier. 
On examination, BCVA was 20/100 in the right eye and no light perception (NLP) in the left eye. Slit-lamp examination of the left eye showed phthisis bulbi, while that of the right eye showed multifocal marginal infiltrates in the superior cornea, corneal guttering, and paracentral corneal perforation in the inferonasal cornea, with normal anterior chamber depth (Fig. 2a). The fundus appearance and intraocular pressures in the right eye were within normal limits.

Laboratory investigations showed an elevated ESR (134 mm/h) and CRP (168 mg/dL). The positive cANCA and rheumatoid factor tests were suggestive of WG. Histopathological examination of the biopsied nasal mucosa showed nonspecific inflammatory changes, and the chest X-ray and renal function measurements revealed no abnormalities.

The patient was treated with intravenous methylprednisolone and $\mathrm{CP}$, which led to amelioration of the ocular inflammation and corneal melt. A tectonic patch graft was performed in this case, as follows: a $12-\mathrm{mm}$ diameter lamellar dissection, using a crescent blade, was created extending to $1.0 \mathrm{~mm}$ from the perforation edge; then a posterior intralamellar corneal patch graft, $2 \mathrm{~mm}$ larger than the perforation, was inserted and secured with buried 10-0 nylon sutures. Finally, an anterior lamellar donor cornea was dissected about $2 \mathrm{~mm}$ onto the sclera, transferred onto the recipient bed, and sutured with 10-0 nylon sutures. Light microscopic examination of the diseased cornea revealed collagen necrosis, as well as infiltration of lymphocytes, eosinophils, epithelioid cells, and multinucleated giant cells (Fig. 2b). Cultures for bacteria and fungi were negative, as were stains for acid-fast bacteria. Oral CP was continued for 8 months. At the 21-month follow-up, vision was 20/200 in the right eye, and the patient's ocular condition remained stable thereafter (Fig. 2c).

\section{Case 6}

A 50-year-old female patient had suffered from redness and pain in the right eye without fever for 5 weeks. Detailed history revealed decreased appetite and moderate weight loss over the previous 3 months. BCVA upon admission was $20 / 40$ in the right eye and 20/30 in the left eye. Slit-lamp examination demonstrated granulomatous necrotizing sclerokeratitis, which involved both cornea and sclera, in the right eye. The conjunctiva was markedly hyperemic, the superior sclera was swollen and inflamed with surface irregularities, and the superior cornea was inflamed across the limbus
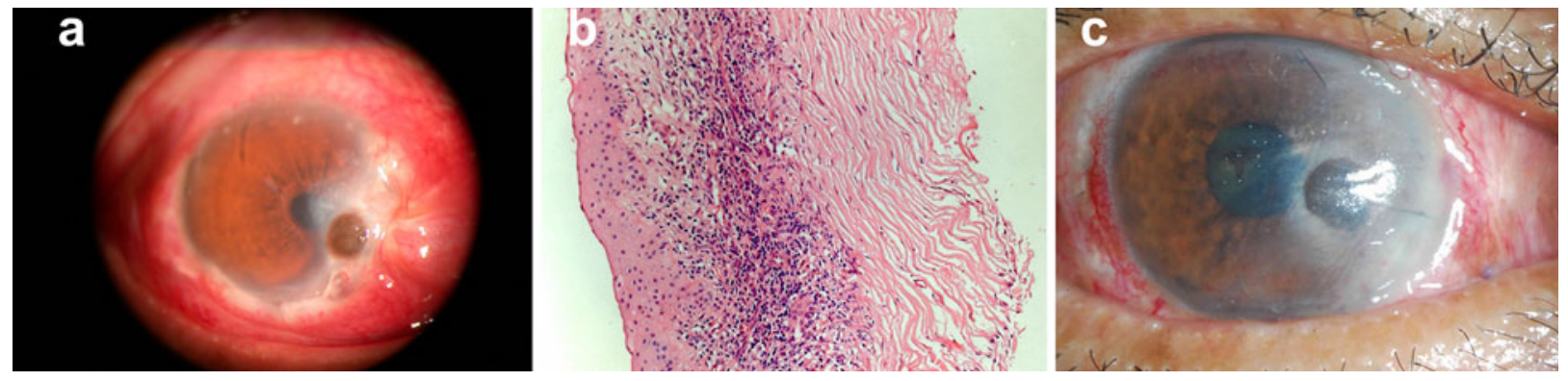

Fig. 2 Patient with necrotizing scleritis and ulcerative peripheral keratitis (case 3). a Circumferential corneal infiltrates, melting, and paracentral corneal perforation of the right eye. $\mathbf{b}$ Corneal biopsy specimen. Note: necrosis of corneal stromal collagen and granulomatous inflammation, with lymphocytes, eosinophils, epithelioid cells, and multinucleated giant cells (magnification $\times 200$; hematoxylineosin stain). c Lamellar corneoscleral graft with intralamellar patch, which was performed to maintain structural integrity 
and showed peripheral corneal ulceration with gutter appearance (Fig. 3a). The anterior chamber was clear, and the fundus appearance and intraocular pressure were normal. Clinical examination of the left eye revealed no abnormalities. Blood studies showed a normal white cell count and an elevated ESR $(74 \mathrm{~mm} / \mathrm{h})$ and CRP $(137 \mathrm{mg} / \mathrm{dL})$. The results of renal function tests were normal. Sinus films were normal, but a chest X-ray showed infiltration in the left lobe, and a nodule and cavitating lesion in the right lobe of the lung (Fig. 3b). A TB skin test was negative, but the cANCA test was positive, raising suspicion of WG. Biopsy of the sclera showed degradation of collagen, inflammatory cells around the capillary and postcapillary venule walls, and infiltrating cells, including lymphocytes, eosinophils, plasma
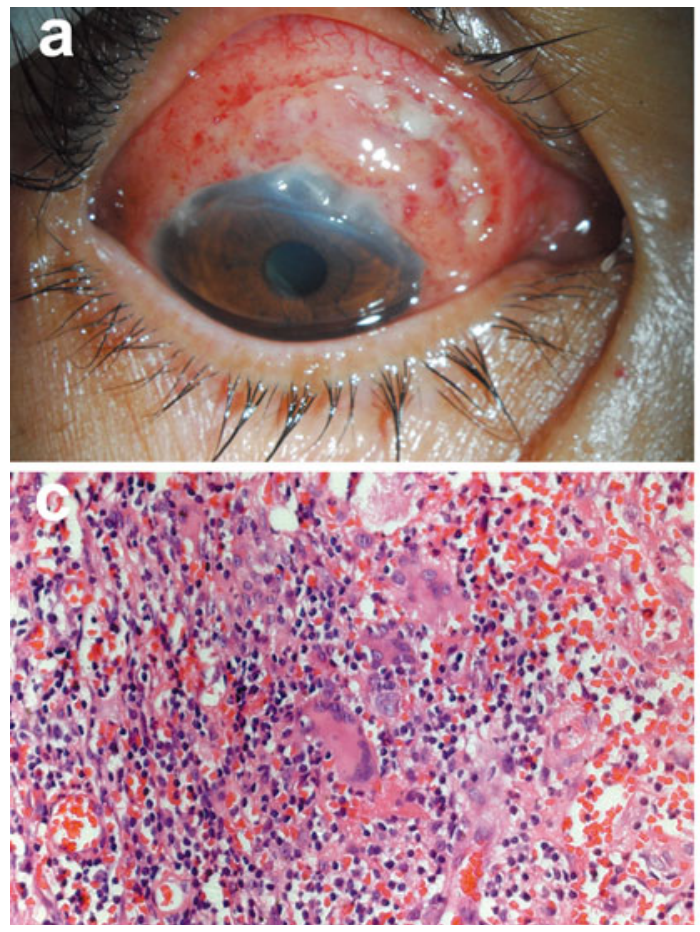

Fig. 3 Montage of clinical and histopathology illustrations (case 6). a Necrotizing scleritis and peripheral ulcerative keratitis. b Chest X-ray showing infiltration in the left lung and a nodule and cavitating lesion in the right lung. c Scleral biopsy, demonstrating giant cells surrounded by

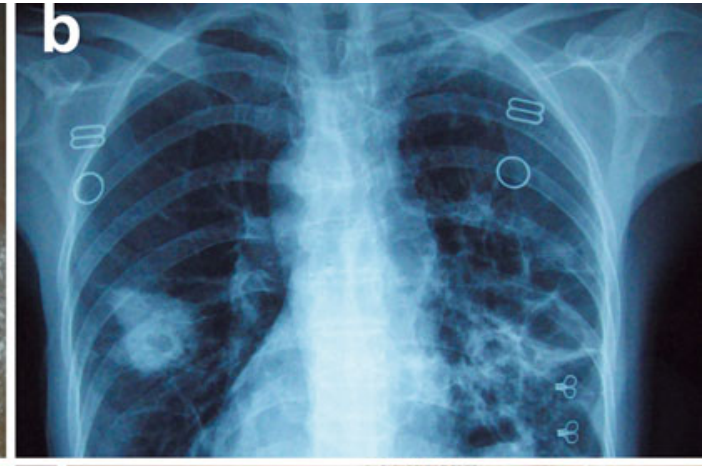

cells, epithelioid cells, and multinucleated giant cells within them (Fig. 3c). The patient was treated with pulse therapy of intravenous methylprednisolone and oral CP, with subsequent subsidence of ocular inflammation and PUK in the right eye (Fig. 3d). Maintenance treatment with oral MTX and reducing oral steroids was continued.

\section{Case 7}

A 30-year-old male patient presented with a 3-month history of redness and soreness in both eyes. The condition was diagnosed as scleritis, but it failed to respond to topical treatment, and the patient was referred to the Department of Ophthalmology in Kashgar's First Hospital for ophthalmic investigation. The patient's medical history included otitis media complicated with

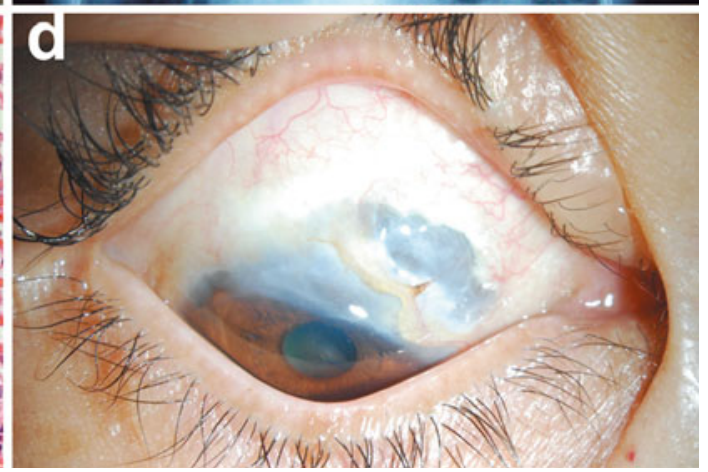

epithelioid cells, lymphocytes, eosinophils, and plasma cells (magnification $\times 400$; hematoxylin-eosin stain). $\mathbf{d}$ Control of the severe necrotizing disease after systemic administration of corticosteroids and cyclophosphamide 

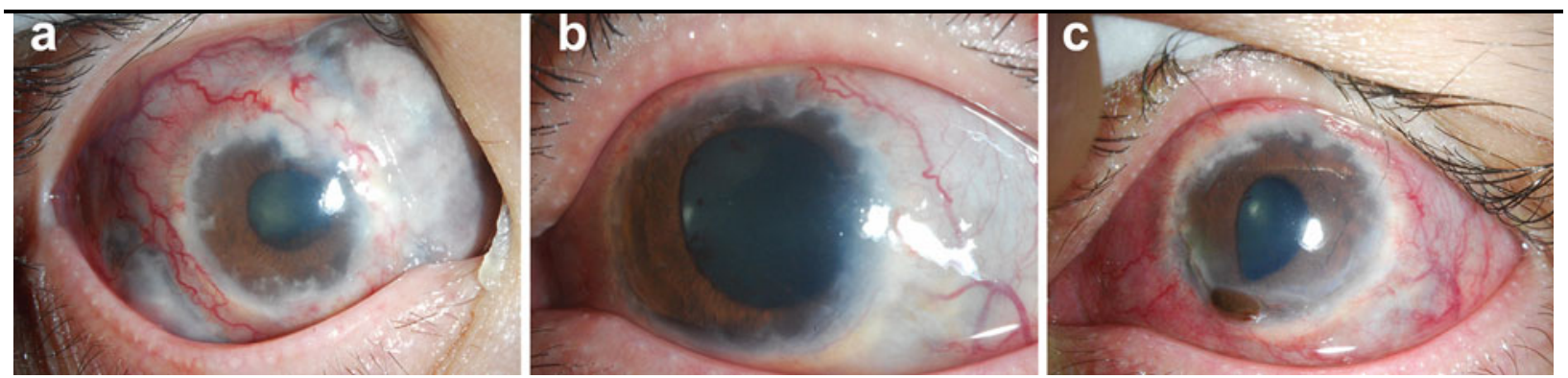

Fig. 4 Clinical pictures of a 30-year-old man with classic Wegener's granulomatosis (case 7). a Slit-lamp examination of the right eye, showing necrotizing scleritis, multiple perilimbal infiltrates, and engorged scleral vessels.

bacterial pneumonia 9 months previously. Six months later, the patient presented with renal failure and arterial hypertension.

Ocular examination disclosed BCVA of 20/100 in both eyes. Slit-lamp examination demonstrated NS and PUK in the right eye (Fig. 4a), and necrotizing anterior scleritis, associated with inferior peripheral corneal gutter and invasion by new vessels from the limbus, in the left eye (Fig. 4b). The anterior chamber was deep, with $1+$ cells in the right eye and no cells in the left eye. The diagnosis of bilateral NS and PUK was made, and because of the clinical and systemic findings, WG was considered.

X-rays revealed mucosal thickening of the right maxillary sinus, and cavitation within areas of pulmonary consolidation. Laboratory tests revealed normocytic normochromic anemia (hemoglobin $7.4 \mathrm{~g} / \mathrm{dL}$ ), and elevated CRP $(43 \mathrm{mg} / \mathrm{dL})$ and ESR $(56 \mathrm{~mm} / \mathrm{h})$. The leukocyte count was slightly elevated $\left(11,200\right.$ cells $\left./ \mathrm{mm}^{3}\right)$, serum creatinine was $4.5 \mathrm{mg} / \mathrm{dL}$, and cANCA titer was positive. Urinalysis was positive for protein, and urinary sediment analysis revealed 6 erythrocytes per high-power field.

The patient was treated with $1,000 \mathrm{mg}$ intravenous CP (every 3 weeks), together with b Necrotizing scleritis and peripheral ulcerative keratitis of the left eye. $\mathbf{c}$ Limbal perforation, which developed in the left eye despite systemic immunosuppression

oral prednisone ( $1 \mathrm{mg} / \mathrm{kg} /$ day), but continued having eye problems. Four weeks after the drug regimen was begun, limbal perforation occurred at the 7 o'clock in the left eye (Fig. 4c); subsequently, more than 9 weeks after limbal perforation, the patient's respiratory function had deteriorated, and serial plain chest radiographs revealed rapidly worsening airspace opacities. The patient was transferred to the intensive care unit and eventually died of respiratory failure.

\section{DISCUSSION}

Wegener's granulomatosis is an insidious disease that affects patients of any age. It consists of a triad: necrotizing granulomatous vasculitis of the upper and the lower respiratory tract, variable degrees of small-vessel vasculitis, and focal necrotizing glomerulonephritis. Classic WG is considered to be a systemic disease; however, a localized form of WG, limited to the upper and lower respiratory tracts, has been reported [9]. Ocular signs and symptoms can be a major component of either classic or limited WG. Ocular manifestations include orbital involvement with proptosis, scleritis, corneal ulceration, uveitis, and nasolacrimal duct obstruction [10]. 
Scleritis has been reported to occur in $16-38 \%$ of patients with WG [11]. NS is strongly associated with systemic disease, and is seen in more than half of the patients with ocular WG [6]. A mortality rate as high as 54\% has been reported in patients with NS who are not undergoing immunosuppressive therapy [12]. NS was found in $86 \%$ of the present patients, and corneal disease was present in most. PUK is typically associated with an underlying systemic disease, most commonly rheumatoid arthritis followed by WG [13]. PUK and scleritis, being associated with rheumatoid arthritis, are manifestations of vasculitis. Scleritis with inflammation, as seen in classic rheumatoid arthritis, does not produce the heaped-up granulomatous lesions seen in systemic vasculitis or Wegener's disease. The episcleral lesion in patients with rheumatoid arthritis is very thin and, therefore, unlikely to become heavily infiltrated and reactive.

It is also probable that the types of inflammatory response in these two diseases are fundamentally different, for a number of reasons. First, the predominant feature in the NS of rheumatoid arthritis is characterized by vascular occlusion, whereas that seen in the vasculitis of WG is characterized by new vessel formation. Second, the corneal changes in rheumatoid arthritis remain confined to the limbus, whereas the inflammatory changes in WG involve the limbal area and progress from sclera to cornea. Third, corneal melt in rheumatoid arthritis usually occurs in patients with long-standing disease; for example, one study found a mean of 19.6 years between the diagnosis of rheumatoid arthritis and the onset of PUK [14], but in WG, corneal melt can occur earlier. Regardless of the underlying condition with which it is associated; however, corneal melt can occur swiftly once inflammation begins within the cornea, and visual loss can ensue within days. Other disease entities also may resemble PUK. Mooren's ulcer has a similar appearance, but no systemic or local associations exist. Terrien's marginal degeneration also presents with peripheral corneal thinning; however, this condition is noninflammatory and in Terrien's, unlike PUK, the epithelium remains intact.

Biopsy and histopathological examination of affected tissues are important for confirming the diagnosis of WG and ruling out other diseases [7]. In the present cases, biopsies of the necrotic sclera revealed a richly polymorphous inflammatory infiltrate, typically featuring plasma cells, lymphocytes, eosinophils, dispersed or clustered epithelioid histiocytes, and multinucleated giant cells. Extravascular granulomas were present in six of the seven cases, and inflammatory microangiopathy was observed in five cases. McCluskey et al. [15] observed that independent areas of necrotizing granulomatous lesions account for much of the tissue destruction, and purely granulomatous disease has been proposed as an early and sometimes the only manifestation of WG [16].

Since WG seldom manifests the classic triad of vasculitis, necrosis, and granulomatous inflammation, the diagnosis must be based on a range of clinical and pathological findings. The cANCA test is specific and sensitive in the diagnosis of WG. All cases in the present series had positive cANCA titers, as measured by enzyme-linked immunosorbent assay, consistent with its validity for this purpose. Unfortunately, the relative rarity of scleritis in general, and of WG-associated scleritis in particular, precludes the examination of a large series of patients. The results of this study demonstrate that cANCA is highly sensitive and specific even for limited WG 
when scleritis is the initial clinical presentation of disease. Because delay in diagnosis and initiation of appropriate treatment in WG can result in vision loss, organ damage, and even death, some have argued for including a positive cANCA titre as a fifth criterion [17]—with nasal or oral inflammation, abnormal chest radiograph, active urine sediment, and positive biopsy-in the modified American College of Rheumatology criteria for the diagnosis of WG.

Because scleritis may be secondary to a systemic disease (as in the cases in this study), and because the inflammatory cells responsible for it originate far from the eye, treatment with systemic corticosteroids and cytotoxic agents is often effective. The condition in the first patient in the present study did not respond to topical dexamethasone for scleritis, but did improve upon treatment with systemic corticosteroids and MTX. Lysis was evident within the scleral graft, despite treatment with systemic corticosteroids and MTX, and finally resolved only when $\mathrm{CP}$ was added. $\mathrm{CP}$ is considered the preferred drug for WG because of its proven efficacy [7], and is associated with fewer disease relapses (although more side effects). There is a strong requirement for a potent but low toxicity treatment on which a patient can possibly be maintained for several years. MTX may be used to maintain remission [18].

Scleral and corneal thinning, which can lead to staphyloma formation, scleral or corneal perforation, and uveal exposure, is not an unusual complication of scleritis and keratoscleritis in WG. Surgical reinforcement of the thinned or perforated sclera or cornea is necessary to prevent prolapse of ocular contents and secondary infection. Various types of allografts have been used to maintain structural integrity in such cases [19-21].
Scleral grafts have many advantages in this situation, as they are readily available from donor eyes, easy to handle, and naturally curved to match the host sclera; they are also avascular and well tolerated, causing little or no inflammatory reaction. Case 1 developed graft lysis within 1 month; this was managed with extensive medical therapy, plus covering by an amniotic membrane graft, which preserved the structural integrity of the eye by facilitating re-epithelialization and vascularization. It is important for surgery to be deferred until medical therapy has controlled the primary disease [22], and for the scleral graft to be covered with a conjunctival flap to prevent its lysis. If the adjacent conjunctiva cannot be mobilized, an amniotic membrane graft may be considered. The amniotic membrane is endowed with anti-inflammatory and epithelialization-promoting properties, it is also immunologically inert and is widely used for ocular surface reconstruction. Amniotic membrane transplantation is only an adjunct to scleral patch grafting, as it reduces inflammation and promotes epithelialization, but it may not provide sufficient structural rigidity.

Surgical resection of conjunctival tissue adjacent to foci of sclerokeratitis has been promoted as a means of decreasing access of inflammatory cells and factors to the peripheral cornea. A retrospective study of 38 patients with sclerokeratitis who underwent adjacent conjunctival biopsy [23] found vasculitis in 20 biopsies. Eighteen of the 20 biopsy-positive patients were treated with chemotherapy; 17 of the 18 had no progression of ulceration. In the two patients not treated with chemotherapy, surgical management stabilized the disease. However, this treatment is controversial, as it is not uniformly agreed that resection alters the course of disease. 


\section{CONCLUSION}

Because of the diversity of the ocular manifestations and the rarity of the disease, WG presents a difficult clinical diagnostic problem. A combination of suggestive biopsy results and elevated cANCA titer is helpful for early diagnosis in cases of limited ophthalmic WG. Immunosuppressive therapy should be instituted after confirming the diagnosis, to prevent progression of the systemic disease. Specific surgical options may be considered in some cases to preserve the integrity of the ocular globe. In order to avoid the associated morbidity and mortality, WG, with the associated NS and PUK, should be managed with aggressive immunosuppression. The ophthalmologist may have an essential role in the early diagnosis and treatment of this disease.

\section{ACKNOWLEDGMENTS}

This study was supported by "Funding of State Key Laboratory of Ophthalmology of China". The authors thank Professor William K. Stell (University of Calgary) for his assistance with medical reviewing of the manuscript. Dr Chen is the guarantor for this article, and takes responsibility for the integrity of the work as a whole.

Conflict of interest. Jianjun $\mathrm{Gu}$, Sheng Zhou, Ruxing Ding, Wumaier Aizezi, Aixin Jiang, and Jiaqi Chen declare no conflict of interest.

Compliance with ethics guidelines. The Ethics Committees of Zhongshan Ophthalmic Center and Kashgar First People's Hospital approved this retrospective study, which has been conducted according to the principles expressed in the Helsinki Declaration of 1975, as revised in 2000 and 2008. The Ethics Committees specifically waived the need for consent.

Open Access. This article is distributed under the terms of the Creative Commons Attribution Noncommercial License which permits any noncommercial use, distribution, and reproduction in any medium, provided the original author(s) and the source are credited.

\section{REFERENCES}

1. Luqmani RA, Bacon PA, Beaman M, et al. Classical versus non-renal Wegener's granulomatosis. Q J Med. 1994;87:161-7.

2. Ahmad I, Lee WC, Nagendran V, Wilson F, Shortridge RT. Localised Wegener's granulomatosis in otolaryngology: a review of six cases. ORL J Otorhinolaryngol Relat Spec. 2000;62:149-55.

3. Rahilly G, Rahilly M. A case of palatal Wegener's granulomatosis. Oral Dis. 2000;6:259-61.

4. Ahmed $\mathrm{M}$, Niffenegger $\mathrm{JH}$, Jakobiec $\mathrm{FA}$, et al. Diagnosis of limited ophthalmic Wegener granulomatosis: distinctive pathologic features with ANCA test confirmation. Int Ophthalmol. 2008;28:35-46.

5. Walton EW. Giant-cell granuloma of the respiratory tract (Wegener's granulomatosis). Br Med J. 1958;2: 265-70.

6. Harper SL, Letko E, Samson CM, et al. Wegener's granulomatosis: the relationship between ocular and systemic disease. J Rheumatol. 2001;28: 1025-32.

7. Langford CA, Sneller MC. Update on the diagnosis and treatment of Wegener's granulomatosis. Adv Intern Med. 2001;46:177-206.

8. Chua J, Lim L. Systemic Wegener's granulomatosis with severe orbito-ocular involvement. Singapore Med J. 2008;49:259-62.

9. Fauci AS, Wolff SM. Wegener's granulomatosis: studies in eighteen patients and a review of the literature. Medicine. 1973;52:535-61. 
10. Haynes BF, Fishman ML, Fauci AS, Wolff SM. The ocular manifestations of Wegener's granulomatosis. Fifteen years' experience and review of the literature. Am J Med. 1977;63:131-41.

11. Thorne JE, Jabs DA. Ocular manifestations of vasculitis. Rheum Dis Clin North Am. 2001;27:761-79.

12. Foster CS, Forstot SL, Wilson LA. Mortality rate in rheumatoid arthritis patients developing necrotizing scleritis or peripheral ulcerative keratitis. Effects of systemic immunosuppression. Ophthalmology. 1984;91:1253-63.

13. Ladas JG, Mondino BJ. Systemic disorders associated with peripheral corneal ulceration. Curr Opin Ophthalmol. 2000;11:468-71.

14. McKibbin M, Isaacs JD, Morrell AJ. Incidence of corneal melting in association with systemic disease in the Yorkshire Region, 1995-7. Br J Ophthalmol. 1999;83:941-3.

15. McCluskey RT, Fienberg R. Vasculitis in primary vasculitides, granulomatoses, and connective tissue diseases. Hum Pathol. 1983;14:305-15.

16. Boudes P. Purely granulomatous Wegener's granulomatosis: a new concept for an old disease. Semin Arthritis Rheum. 1990;19:365-70.
17. Leavitt RY, Fauci AS, Bloch DA, et al. The American College of Rheumatology 1990 criteria for the classification of Wegener's granulomatosis. Arthritis Rheum. 1990;33:1101-7.

18. White ES, Lynch JP. Pharmacological therapy for Wegener's granulomatosis. Drugs. 2006; 66:1209-28.

19. Sainz de la Maza M, Tauber J, Foster CS. Scleral grafting for necrotizing scleritis. Ophthalmology. 1989;96:306-10.

20. Koenig SB, Sanitato JJ, Kaufman HE. Long-term follow-up study of scleroplasty using autogenous periosteum. Cornea. 1990;9:139-43.

21. Torchia RT, Dunn RE, Pease PJ. Fascia lata grafting in scleromalacia perforans with lamellar cornealscleral dissection. Am J Ophthalmol. 1968;66: 705-9.

22. Nguyen QD, Foster CS. Scleral patch graft in the management of necrotizing scleritis. Int Ophthalmol Clin. 1999;39:109-31.

23. Tauber J, Sainz de la Maza M, Hoang-Xuan T, Foster CS. An analysis of therapeutic decision making regarding immunosuppressive chemotherapy for peripheral ulcerative keratitis. Cornea. 1990;9: 66-73. 